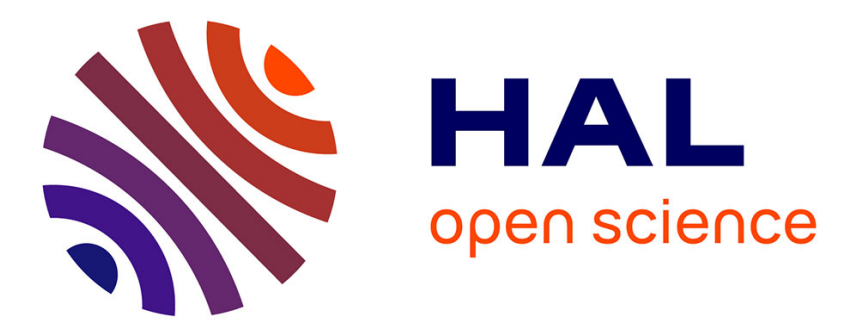

\title{
Highlighting and Reducing the Impact of Negative Aging Stereotypes During Older Adults' Cognitive Testing
}

Marie Mazerolle, Isabelle Regner, François Rigalleau, Pascal Huguet

\section{To cite this version:}

Marie Mazerolle, Isabelle Regner, François Rigalleau, Pascal Huguet. Highlighting and Reducing the Impact of Negative Aging Stereotypes During Older Adults' Cognitive Testing. Journal of visualized experiments: JoVE, 2020, 155, pp.e59922. 10.3791/59922 . hal-02458675

\section{HAL Id: hal-02458675 \\ https://hal.science/hal-02458675}

Submitted on 28 Jan 2020

HAL is a multi-disciplinary open access archive for the deposit and dissemination of scientific research documents, whether they are published or not. The documents may come from teaching and research institutions in France or abroad, or from public or private research centers.
L'archive ouverte pluridisciplinaire HAL, est destinée au dépôt et à la diffusion de documents scientifiques de niveau recherche, publiés ou non, émanant des établissements d'enseignement et de recherche français ou étrangers, des laboratoires publics ou privés. 
TITLE:

Highlighting and Reducing the Impact of Negative Aging Stereotypes During Older Adults' Cognitive Testing

AUTHORS AND AFFILIATIONS:

Marie Mazerolle ${ }^{1}$, Isabelle Régner ${ }^{2}$, François Rigalleau ${ }^{3}$, Pascal Huguet ${ }^{4}$

${ }^{1}$ Department and Laboratory of Psychology, MSHE, University of Burgundy Franche-Comté, Besançon, France

$10 \quad{ }^{2}$ Aix-Marseille University, CNRS, LPC, Marseille, France

113 Department of Psychology, University of Poitiers, CNRS, Poitiers, France

12 4University of Clermont Auvergne, CNRS, LAPSCO, Clermont-Ferrand, France

Corresponding author:

15 Isabelle Régner (isabelle.regner@univ-amu.fr)

Email addresses of co-authors:

Marie Mazerolle (marie.mazerolle@univ-fcomte.fr)

François Rigalleau (francois.rigalleau@univ-poitiers.fr)

Pascal Huguet (pascal.huguet@uca.fr)

\section{KEYWORDS:}

behavior, stereotype threat, aging, memory, neuropsychological testing, dementia, social cognition

\section{SUMMARY:}

Here, we present a protocol designed to show how negative aging stereotypes can impair memory performance of older adults during cognitive testing and how to reduce this deleterious effect. This method can help older people to perform at an optimal level during testing in both lab studies and clinical settings.

\section{ABSTRACT:}

As life expectancy increases, aging has become a major health challenge, resulting in a huge effort to better discriminate between normal and pathological cognitive decline. It is thus essential that cognitive tests and their administration are as fair as possible. However, an important source of bias during cognitive testing comes from negative aging stereotypes that can impair the memory performance of older adults and inflate age differences on cognitive tasks. The fear of confirming negative aging stereotypes creates an extra pressure among older adults which interferes with their intellectual functioning and leads them to perform below their true abilities. Here, we present a protocol that highlights simple but efficient interventions to alleviate this age-based stereotype threat effect. The first study showed that simply informing older participants about the presence of younger participants (threat condition) led older adults to underperform on a standardized memory test compared with younger 
as age-fair (reduced-threat condition). The second study replicated these findings on short cognitive tests used to screen for predementia in clinical settings and showed that teaching older adults about stereotype threat inoculated them against its effects. These results provide useful recommendations about how to improve older adults' memory assessment both in lab studies and in clinical settings.

\section{INTRODUCTION:}

A growing field of laboratory research in social cognition conducted in the healthy population has demonstrated that members of groups whose abilities are negatively stereotyped typically underperform when the negative stereotypes are made relevant to the performance at hand, a phenomenon called stereotype threat (ST). In addition to the normal anxiety associated with taking cognitive tests, the fear of confirming negative stereotypes creates extra pressure that may interfere with cognitive functioning and lead to perform below one's abilities ${ }^{1,2}$. Many findings demonstrate that negative aging stereotypes (e.g., the culturally shared beliefs that aging inescapably causes severe cognitive decline and diseases such as Alzheimer's disease [AD]) contribute, at least in part, to the differences classically observed in the healthy population between younger and older adults in memory tasks $\mathrm{s}^{3-5}$. Without denying the impact of aging on cognitive functioning, research clearly demonstrates that age-related stereotypes are powerful enough to artificially decrease older adults' performance on memory tests.

Detrimental age-based ST effects are readily observable and fairly easy to produce with instructional manipulations ${ }^{6}$, such as simply emphasizing the memory component of the test ${ }^{7-9}$, highlighting differences in performance between young and older adults ${ }^{10,11}$, or implicitly activating negative aging stereotypes ${ }^{12,13}$. Given the results obtained in laboratory studies, it is very likely that negative aging stereotypes also permeate, at least implicitly, the standard neuropsychological testing settings during the screening for pre-dementia. Indeed, because of the lengthening of life expectancy, more and more people are concerned with the possibility of getting $A D$ or other forms of dementia. Importantly, false-positive errors are quite frequent in the diagnosis of prodromal state of $A D^{14}$, which could be explained, at least in part, by transitory impaired performances in older adults due to the age-based ST phenomenon ${ }^{15}$.

For these reasons, it is important to provide efficient methods to deactivate the influence of negative aging stereotypes and thereby help older people to perform at their maximum during memory assessment in general and during neuropsychological testing specifically. Some methods, such as de-emphasizing the memory component of the test (e.g., characterizing the task as a vocabulary test), have already proved efficient to eliminate ST effects in older adults on explicit memory tests taken in the context of lab studies ${ }^{7,9,16}$. However, such instructions are not compatible with the ecological clinical context of the neuropsychological testing, in which older adults come to have their memory abilities assessed. The goal of our articles is to present two methods likely to alleviate age-based ST effects among older adults, either in the lab or in the clinical contexts. The first one, especially suited for the lab context, consists in telling older adults that performance on the ongoing memory tests usually does not differ between younger and older adults (i.e., age-fair instructions). The second method, which can be implemented in 
impact of aging stereotypes, which may help them reappraise the situation, reduce evaluative

\section{PROTOCOL:}

The present research was carried out in accordance with French standards, each participant provided informed consent and procedures were consistent with the guidelines of the American Psychological Association.

\section{Highlighting and reducing age-based stereotype threat on a lab memory test}

\subsection{Participants screening}

1.1.1. Recruit participants in the desired age range (e.g., younger participants: $18-35$ years old; older participants: 60-85 years old) for a study on general mental abilities and/or the impact of emotions on various cognitive tasks and questionnaires.

1.1.2. Ensure that participants are native speakers, have normal or corrected-to-normal vision and hearing, are free of neurological and psychiatric disorders, and have no current depression (Geriatric Depression Scale ${ }^{17}$ ) nor abnormal anxiety (State-Trait Anxiety Inventory ${ }^{18}$ ).

1.1.3. Screen older participants for cognitive impairment using the Mini-Mental State Examination (MMSE) ${ }^{19}$. Include older adults who meet or exceed a cutoff score corresponding to his/her age and educational level ${ }^{20}$, and who live at home.

\subsection{Conditions and experimental design}

1.2.1. Test each participant individually at home or in local community associations' premises by an experimenter trained at neuropsychological testing.

\subsubsection{Run the study as a single session, but inform participants there are two separate studies.}

1.2.3. Use the "first study" as a baseline measure of participants' working memory capacity. In the "first study", minimize evaluative pressure, informing participants they will perform a cognitive task currently under elaboration. Encourage participants to do their very best ${ }^{21}$.

1.2.4. Use the "second study" to evaluate the impact of stereotype threat on participants' working memory capacity. That is, inform participants that they are going to perform a fully validated and diagnostic test of memory capacity.

1.2.4.1. Randomly assign participants to one of two conditions (threat versus reduced-threat conditions). 
1.2.4.2. In the "threat condition", simply tell participant that both younger and older adults are participating in the study.

1.2.4.3. In the "reduced threat condition", tell participants that both younger and older adults are participating in the study but add that younger and older adults usually obtain the same performances on the ongoing test.

\subsection{Memory tests and procedure}

1.3.1. Use a validated memory test that is typically used in cognitive lab studies. Because ST effects typically occur on difficult tasks, use a difficult test, such as the reading span test ${ }^{23}$.

1.3.2. Use two alternative versions of the reading span test (one for the "first study" and the other one for the "second" study), with different sentences but match them in number of words and in length, frequency, and number of syllables of the last word.

1.3.3. Use a reading span test that contains 42 sentences: 12 series of two to five sentences 151 with 3 series per length.

1.3.4. Present one sentence at a time and instruct participant to read it, at his/her own pace, while memorizing the last word of the sentence.

\subsubsection{Ask participant not to interrupt his reading and not to pause between two sentences.}

1.3.6. Have participant to recall the words immediately after he/she finished reading the last sentence of the block considered.

1.3.7. Do not require participants' recall being serial but constrain them not to start by recalling the final word of the last sentence read. Do not limit the recall time and invite participants to find as many words as possible.

1.3.8. Do not inform participants of their errors, and do not give them a second chance to answer.

1.3.9. Compute the final score of each participant using the mean proportion of correctly recalled words across the 12 series.

2. Highlighting and reducing age-based stereotype threat on short cognitive tests used to screen for predementia in clinical settings

2.1. Participants screening 
2.1.1. Recruit older participants aged 60 to 85 for a study on general mental abilities and/or the impact of emotions on various cognitive tasks and questionnaires.

2.1.2. Ensure that participants live at home, have no history of significant trauma nor chronic illness, have normal or corrected-to-normal vision and hearing, have no current depression (Geriatric Depression Scale ${ }^{17}$ ) nor abnormal anxiety (State-Trait Anxiety Inventory ${ }^{18}$ ).

\subsection{Conditions and experimental design}

2.2.1. Test each participant individually at home or in local community associations' premises by an experimenter trained at neuropsychological testing.

2.2.2. Have participants taking two short cognitive tests used to screen for predementia (or mild cognitive impairment $[\mathrm{MCl}])$ : the $\mathrm{MMSE}^{19}$ and the Montreal Cognitive Assessment $\left(\mathrm{MoCA}^{24}\right)$ in a counterbalanced order.

2.2.3. Randomly assign participants to one of the two order (MMSE then MoCA or MoCA then MMSE).

2.2.4. Before taking the first test, randomly assign participants to one of two threat conditions (threat versus reduced-threat conditions).

2.2.5. In the "threat condition", inform participants that both younger and older adults are taking part in the study about memory capacity.

2.2.6. In the "reduced threat condition", inform participants that both younger and older adults are taking part in the study about memory capacity but add that younger and older adults typically obtain the same performances on the ongoing tests.

2.2.7. Before taking the second test, use an educational intervention to debrief all participants (regardless of the threat condition they were previously assigned) about age-based stereotype threat. Tell participants that "Numerous studies demonstrated that negative age-related beliefs, especially about memory capacity decline, trigger anxiety, which in turn prevents older adults from performing optimally. Actually, it is well-known that performance on memory tests may not accurately reflect people's true abilities, because performance can be affected by the testing context. In other words, people's negative reputation in an ability domain may impair their performance on tests that assess this stereotyped ability, for example when older adults complete a memory test. Therefore, if you feel anxious during the subsequent memory test, this does not mean that you are unable to complete the tests. It rather probably means that your feelings are affected by the general negative reputation of older adults regarding memory."

NOTE: See Figure 2 for a visual depiction of the educational intervention; full details on this research were published in Mazerolle et al. ${ }^{25}$. 
2.3.1. Administrate the 8 subtests of the $\mathrm{MMSE}^{19}$ (orientation, registration, attention and calculation, recall, language, copying) to evaluate memory, orientation to time and place, attention and executive functioning, language and visuospatial abilities. Follow Folstein, Folstein and McHugh's administration guidelines ${ }^{19}$ and compute the final score adding the scores of every subtest. Obtain a final score rated on 30-point scale.

2.3.2. Administrate the 8 subtests of the $\mathrm{MoCA}^{24}$ (visuospatial/executive, naming, memory, attention, language, abstraction, delayed recall, orientation) to evaluate memory, orientation to time and place, attention and executive functioning, language and visuospatial abilities. Follow administration guidelines available at www.mocatest.org and compute the final score adding the scores of every subtest. Obtain a final score rated on 30-point scale.

\section{REPRESENTATIVE RESULTS:}

We addressed the hypothesis that stereotype threat impairs the working memory performance of older adults and that this effect can be reduced or eliminated by a simple instruction. The expected interaction between age group and threat instructions was significant, $F(1,214)=$ 4.85, $p<0.03, \eta_{p}{ }^{2}=0.02$, and is depicted in Figure 3. In the threat condition, older participants underperformed (mean $[M]=0.73$, standard error $[S E]=0.01$ ) relative to younger participants $(M=0.78, S E=0.01), F(1,214)=12.87, p<0.001, \eta_{p}^{2}=0.06$, whereas the two age groups performed equally well in the reduced-threat condition $(F<1)$. Moreover, older participants in the reduced-threat condition obtained a better working memory score $(M=0.77, S E=0.01)$ relative to those in the threat condition $(M=0.73, S E=0.01), F(1,214)=9.42, p<0.002, \eta_{\mathrm{p}}^{2}=$ 0.04 . The performance of younger participants did not differ across the two conditions (threat condition: $M=0.78, S E=0.01$; reduced-threat condition: $M=0.78, S E=0.01 ; F<1$ ). More details about this research can be found in Mazerolle et al. ${ }^{22}$. These findings highlight the deleterious impact of very subtle cues in the environment (e.g., mentioning the presence of younger adults in a study about memory) on older adults' performance on a laboratory test. Because stereotype threat seems to be powerful enough to create significant differences between younger and older adults, we went further and tested its impact on brief cognitive tests classically used by primary care clinicians for the diagnosis of predementia.

We tested the impact of stereotype threat on older adults' performance on two brief cognitive tests used to screen for predementia states: the MoCA and the MMSE. As shown in Figure 4 (left panel), during test 1 (before debriefing), participants' performance was higher in the reduced-threat condition $(M=28.50 ; S E=0.47)$ than in the threat condition $(M=26.40 ; S E=$ 0.43 ) on the MMSE, $F(1,76)=12.506, p=0.001, \eta_{p}{ }^{2}=0.141$. The same pattern occurred on MoCA, with participants underperforming in the threat condition $(M=24.80 ; S E=0.53)$ as compared to the reduced-threat condition $(M=27.45$; $S E=0.53), F(1,76)=10.153, p=0.002$, $\left.\eta_{\mathrm{p}}{ }^{2}=0.118\right)$. These results show that the performances of older adults on short cognitive tests 
Because these tests are used to screen for predementia state, we also examined the proportion of participants meeting the clinical criteria of $\mathrm{MCl}$ on MoCA and MMSE (using a cutoff at 26 for both tests). During test 1 , stereotype threat was powerful enough on the MoCA to result in $50 \%$ of our sample performing under the cutoff score of $26 / 30$ in the threat condition, versus $15 \%$ in the reduced-threat condition ( $p=0.041$, Fischer's exact test). The same pattern occurred on MMSE, with $30 \%$ of the participants scoring below 26 in the threat condition, against $5 \%$ in reduced-threat condition ( $p=0.038$, Fisher's exact test). More information about this study can be found in Mazerolle et al. ${ }^{25}$.

We also hypothesized that an educational intervention (debriefing) consisting in informing older adults about the stereotype threat phenomenon and alleviating the evaluative pressure of the test, would reduce the impact of negative aging stereotypes on their performance. As shown in Figure 4 (right panel), after the educational intervention (debriefing), older adults performed equally well on test 2 (be it MoCA or MMSE), regardless of the administration condition of their previous test 1 , threat or reduced-threat. In other words, lower performance of participants at test 1 due to threat instructions was restored at test 2 thanks to the educational intervention. More details about these findings can be found in Mazerolle et al. ${ }^{25}$.

FIGURE LEGENDS:

Figure 1: Visual depiction of the procedure to test age-based stereotype threat on a lab memory test.

Figure 2: Visual depiction of the educational intervention to reduce age-based stereotype threat effects.

Figure 3: Reading span score (adjusted for covariates) as a function of instructions condition and age group. Error bars indicate standard errors of the mean. This figure has been modified from Mazerolle et al. ${ }^{22}$.

Figure 4: MMSE and MoCA scores as a function of threat conditions before debriefing and after debriefing participants about the impact of stereotype threat. Error bars represent standard errors of the mean. This figure has been modified from Mazerolle et al. ${ }^{25}$.

\section{DISCUSSION:}

The present studies show that stereotype threat, a neglected source of stress in many testing situations, can lead older adults to perform below their true abilities on memory tests. The method presented here highlights the crucial importance of the instructions given to participants and patients before testing memory. Simply mentioning that younger adults are taking part in the study (without mentioning any expected age-related differences in performance) is sufficient to inflate by $40 \%$ (MMSE and MoCA averaged) the number of older adults meeting clinical criteria for predementia on short cognitive tests. The present findings also showed that presenting the memory tests as age fair or explaining to older adults the negative impact of aging stereotypes on their performances are two efficient strategies to help 
them resist to age-based stereotype threat. Taken together, these results highlight the critical importance of taking into account stereotype treat effects when assessing older adults' memory using short cognitive tests, especially given the current pressure on general practitioners to participate in the screening for predementia ${ }^{26,27}$. It is also important for experimental studies about cognitive aging because short cognitive tests are used to exclude participants who are suspected to show predementia symptoms only because they scored below a cutoff.

Some of the critical aspects of the method deserve particular attention. A typical mistake when one discovers stereotype threat research ${ }^{28}$ consists in assuming that a stereotype threat condition requires the implementation of an extra pressure that does not exist in conventional real-life testing, and that standard real-life testing instructions can be used to operationalize a no-stereotype threat control condition. Actually, it is quite the contrary: The standard real-life testing settings are likely to induce stereotype threat, either implicitly or explicitly, because of the words used to present the tests and/or any environmental cues that are related to negative aging stereotypes (e.g., the presence of flyers or posters on Alzheimer's disease or other dementia). It is much more difficult to find a way to eliminate stereotype threat in a testing situation. Therefore, any modification to the instructions that have been found efficient to reduce stereotype threat should be carefully considered to avoid the belief that one is comparing a threat and a reduced-threat condition while, in fact, one is comparing two stereotype threat conditions.

Although the present findings were obtained on lab memory tests and short cognitive tests, they raise the question of whether age-based stereotype threat effects can also affect older patients' performances on complete neuropsychological batteries used in memory clinics for the diagnosis of $\mathrm{MCl}$, the prodromal stage of $\mathrm{AD}$. In our view, it seems very likely that age-based stereotype threat effects contribute, at least in part, to the $53 \%$ of false positive errors observed in the diagnosis of $\mathrm{MCl}$. Without denying that aging may be associated with cognitive decline and neurodegenerative diseases such as $\mathrm{MCl}$ or $A D$ for many people, our findings suggest paying special attention to the influence of negative aging stereotypes that have been largely overlooked in the context of neuropsychological testing.

Several individual factors can make older adults more or less vulnerable to age-based stereotype threat effects. As indicated by a recent review ${ }^{5}$, these moderators include older adults' level of education, physical and psychological health, subjective age, stigma consciousness, domain identification (i.e., importance attached to memory), and memory selfefficacy. Future research, especially those conducted within the clinical setting where a diagnosis is requested, should thus consider some of these factors to provide fairer evaluation of patients' memory abilities.

Our experimental design offers new recommendations to healthcare professionals to improve the accuracy of predementia diagnosis. Characterizing the memory tests as age fair or informing older adults about the age-based stereotype threat phenomenon are two simple and easily implementable instructions likely to help older people to perform at an optimal level 
during neuropsychological testing. These recommendations may help medical staff to deliver more accurate and potentially less threatening information to the patients and/or their family (thereby significantly improving their well-being and quality of life). It should be noted, however, that these recommendations may need to be adapted to consider other types of threat that could be simultaneously triggered by the clinical context (e.g., the specific threat of $A D$ as a dramatic disease ${ }^{29}$, the threat of being at hospital ${ }^{30}$, and the threat of the white coat ${ }^{31}$ ).

\section{ACKNOWLEDGMENTS:}

Part of this work was supported by Plan Alzheimer Foundation on a Humanities and Social Sciences grant (AAP SHS 2013: "Sociocognitive aspects of Alzheimer disease" to F. Rigalleau and M. Mazerolle).

\section{DISCLOSURES:}

The authors have nothing to disclose.

\section{REFERENCES:}

1. Steele, C. M., Aronson, J. Stereotype threat and the intellectual test performance of African Americans. Journal of Personality and Social Psychology. 69 (5), 797-811 (1995).

2. Steele, C. M. A threat in the air: How stereotypes shape intellectual identity and performance. American Psychologist. 52, 613-629 (1997).

3. Barber, S. J. An examination of age-based stereotype threat about cognitive decline: Implications for stereotype-threat research and theory development. Perspectives on Psychological Science. 12 (1), 62-90 (2017).

4. Chasteen, A. L., Kang, S. K., Remedios, J. D. Aging and stereotype threat: Development, process, and interventions. In Stereotype threat: Theory, process, and application. Edited by Inzlicht M., Schmader T., 202-216, Oxford University Press. New York, NY (2012).

5. Lamont, R. A, Swift, H. J., Abrams, D. A. Review and Meta-Analysis of Age-Based Stereotype Threat: Negative Stereotypes, Not Facts, Do the Damage. Psychology and Aging. 30 (1), 180-193 (2015).

6. Haslam, C. et al. "When the age is in, the wit is out": Age related self-categorization and deficit expectations reduce performance on clinical tests used in dementia assessment. Psychology and Aging. 27, 778-784 (2012).

7. Desrichard, O., Köpetz, C. A threat in the elder: The impact of task-instructions, self-efficacy and performance expectations on memory performance in the elderly. European Journal of Social Psychology. 35, 537-552 (2005).

8. Kang, S. K., Chasteen, A. L. The moderating role of age-group identification and perceived threat on stereotype threat among older adults. The International Journal of Aging and Human Development. 69, 201-220 (2009). 9. Rahhal, T. A., Hasher, L., Colcombe, S. J. Instructional manipulations and age differences in memory: Now you see them, now you don't. Psychology and Aging. 16, 697-706 (2001). 10. Hess, T. M., Auman, C., Colcombe, S. J., Rahhal, T. A. The Impact of Stereotype Threat on Age Differences in Memory Performance. The Journals of Gerontology Series B: Psychological Sciences and Social Sciences. 58 (1), P3-P11 (2003). 
11. Hess, T. M., Hinson, J. T. Age-related variation in the influences of aging stereotypes on memory in adulthood. Psychology and Aging. 21 (3), 621-625 (2006). 12. Levy, B. Improving memory in old age through implicit self-stereotyping. Journal of Personality and Social Psychology. 71, 1092-1107 (1996). 13. Stein, R., Blanchard-Fields, F., Hertzog, C. The effects of age-stereotype priming on the memory performance of older adults. Experimental Aging Research. 28 (2), 169-181 (2002). 14. Sachdev, P. S. et al.; Sydney Memory, Ageing Study Team. Factors predicting reversion from mild cognitive impairment to normal cognitive functioning: a population-based study. PloS One. 8, e59649 (2013).

15. Régner, I. et al. Aging stereotypes must be taken into account for the diagnosis of prodromal and early Alzheimer's disease. Alzheimer Disease \& Associated Disorders - An International Journal. 30 (1), 77-79 (2016).

16. Chasteen, A. L., Bhattacharyya, S., Horhota, M., Tam, R., Hasher, L. How feelings of stereotype threat influence older adults' memory performance. Experimental Aging Research. 31 (3), 235-260 (2005). 17. Yesavage, J. A. et al. Development and validation of a geriatric depression screening scale: a preliminary report. Journal of Psychiatric Research. 17 (1), 37-49 (1982). 18. Spielberger, C. D. Test Anxiety Inventory. The Corsini Encyclopedia of Psychology. John Wiley \& Sons, Inc. Hoboken, NJ (2010). 19. Folstein, M. F., Folstein, S. E., McHugh, P. R. "Mini-mental state." Journal of Psychiatric Research. 12 (3), 189-198 (1975). 20. Crum, R. M., Anthony, J. C., Bassett, S. S., Folstein, M. F. Population-based norms for the Mini-Mental State Examination by age and educational level. The Journal of the American Medical Association. 269 (18), 2386-2391 (1993). 21. Gimmig, D., Huguet, P., Caverni, J-P., Cury, F. Choking under pressure and working-memory capacity: When performance pressure reduces fluid intelligence. Psychonomic Bulletin \& Review. 13, 1005-1010 (2006).

22. Mazerolle, M., Régner, I., Morisset, P., Rigalleau, F., Huguet, P. Stereotype Threat Strengthens Automatic Recall and Undermines Controlled Processes in the Elderly. Psychological Science. 23, 723-727 (2012). 23. Daneman, M., Carpenter, P. A. Individual differences in working memory and reading. Journal of Verbal Learning and Verbal Behavior. 19, 450-466 (1980). 24. Nasreddine, Z. S. et al. The Montreal Cognitive Assessment, MoCA: A brief screening tool for mild cognitive impairment. Journal of the American Geriatrics Society. 53, 695-699 (2005). 25. Mazerolle, M. et al. Negative Aging Stereotypes Impair Performance on Brief Cognitive Tests Used to Screen for Predementia. Journals of Gerontology, Series B: Psychological Sciences and Social Sciences. 72 (6), 932-936 (2017). 26. Brown, J. The use and misuse of short cognitive tests in the diagnosis of dementia. Journal of Neurology, Neurosurgery, and Psychiatry. 86, 680-685 (2015). dementia: not evidence based and ignores the harms of diagnosis. BMJ. 347, f5125 (2013). 28. Steele, C.M., Davies, P.G. Stereotype Threat and Employment Testing: A Commentary. Human Performance. 16 (3), 311-326 (2003). 
438 29. Scholl, J. M., Sabat, S. R. Stereotypes, stereotype threat and ageing: implications for the 439 understanding and treatment of people with Alzheimer's disease. Ageing \& Society. 28 (1), 440 103-130 (2008).

441 30. Suhr, J. A., Gunstad, J. "Diagnosis threat": The effect of negative expectations on cognitive 442 performance in head injury. Journal of Clinical and Experimental Neuropsychology. 24 (4), 443 448-457 (2002).

444 31. Schlemmer, M., Desrichard, O. Is Medical Environment Detrimental to Memory? A Test of A 445 White Coat Effect on Older People's Memory Performance. Clinical Gerontologist. 41 (1), 77-81 446 (2018). 